\title{
PERDAS FETAIS E NATIMORTALIDADE NO MUNICÍPIO DE RIBEIRÃO PRETO-SP, BRASIL, 1991 E 1992
}

FETAL MORTALITY IN RIBEIRÃO PRETO CITY, BRAZIL, 1991 AND 1992

\author{
Renato Sardas $^{1}$ \& Amábile Rodrigues Xavier Manço²
}

\begin{abstract}
${ }^{1}$ Bolsista de Iniciação Científica do CNPq; ${ }^{2}$ Docente do Departamento de Medicina Social da Faculdade de Medicina de Ribeirão Preto da Universidade de São Paulo.

CorRespondÊncia: Profa.Dra. Amábile R. X. Manço - Departamento de Medicina Social - Faculdade de Medicina de Ribeirão Preto da Universidade de São Paulo - Campus Universitário - CEP: 14048-900 - Ribeirão Preto - SP - Brasil. Fax: (016) 633-5839.
\end{abstract}

SARDAS R \& MANÇO ARX. Perdas fetais e natimortalidade no Município de Ribeirão Preto-SP, Brasil, 1991 e 1992. Medicina, Ribeirão Preto, 30: 508-513, out./dez. 1997.

RESUMO: No Município de Ribeirão Preto ocorreram 299 perdas fetais nos anos de 1991/92, sendo 165, em 1991, e 134, em 92, dos quais 148 eram masculinos, 134 femininos, 21 de sexo ignorado e 15 não declarados.

Os óbitos fetais provenientes de outros municípios perfizeram 39,8\% deste total. Em 103 Declarações de Óbito, o peso ao nascer não foi declarado.

Com relação à duração de gestação, 142 casos $(47,5 \%)$ tinham 28 ou + semanas, $52(17,4 \%)$ situavam-se entre 20 e 27 semanas, em 105 casos (35,3\%) a informação estava em branco ou assinalada como ignorada.

A gestação única foi apontada em $46,8 \%$ dos casos e não declarada em $46,5 \%$.

A hipoxia intra-uterina foi apontada como causa básica de morte, em $41,8 \%$ dos casos.

UNITERMOS: Morte Fetal. Mortalidade Fetal.

\section{INTRODUÇÃO}

A morte fetal, medida comumente por meio do coeficiente de natimortalidade, pode ser usada como um indicador de saúde que reflete as condições relativas à gestação, qualidade de consultas pré-natais e assistência ao parto, assim como o nível econômico-cultural das gestantes, suas condições de trabalho, enfim, a qualidade de vida e a situação de saúde.

Entende-se por óbito fetal, perda fetal ou morte fetal "a morte de um produto de concepção antes da expulsão completa do corpo da mãe, independente do tempo de gestação". A morte do feto é caracterizada pela inexistência, depois da separação do corpo da mãe, de qualquer sinal de vida, seja respiração, batimentos cardíacos, pulsação do cordão umbilical ou movimentos efetivos dos músculos de contração voluntária ${ }^{(1)}$.
Embora diversos pesquisadores ${ }^{(2,3)}$, em diferentes regiões do país, tenham demonstrado que o mal preenchimento da Declaração de Óbito (D.O) seja prática disseminada em nosso meio, é o instrumento freqüentemente utilizado para colher dados, uma vez que se trata de um documento exigido por lei, para a realização do sepultamento.

A legislação em vigor reza a obrigatoriedade do registro de nascidos mortos com idade gestacional igual ou superior a 28 semanas $^{(4)}$. Há uma recomendação, por parte de pesquisadores, para se fazer também o registro das perdas fetais que ocorrem entre 20 e 27 semanas de gestação, no sentido de recuperar estes dados. Assim, o modelo de Declaração de Óbito em uso no país, nos anos de 1991 e 1992, tinham campos separados para idade gestacional menor de $20 \mathrm{se}-$ manas, 20 a 27 e outro para 28 ou mais. No entanto, 
em termos de registro, todas as Declarações de Óbito Fetal são feitas como se se tratasse de natimortos.

$\mathrm{O}$ estudo da natimortalidade, embora reconhecidamente fundamental no planejamento da saúde populacional, tem sido pouco investigado em nosso meio.

No presente trabalho, desenvolvido com dados de Declarações de Óbitos referentes a óbitos fetais registrados em Ribeirão Preto, foram estudadas as variáveis maternogestacionais e fetais em busca da detecção de dados que retratassem a realidade de natimortalidade do município, com os seguintes objetivos:

1 - quantificar os óbitos fetais do município, segundo procedência;

2 - relacionar algumas variáveis maternogestacionais com as perdas fetais;

\section{MATERIAL E MÉTODOS}

O Município de Ribeirão Preto, por dispor de amplos recursos médico-hospitalares, destacando-se o Hospital das Clínicas da FMRP-USP, é um ponto de convergência para os que necessitam de assistência médica. Muitos pacientes atendidos procedem de outros municípios da região ou até de outros estados brasileiros.

O Município de Ribeirão Preto conta com quatro Cartórios de Registro Civil, três urbanos e um rural. As Declarações de Óbito registradas são xerocopiadas pelo Sistema de Coleta e Análise de Estatísticas Vitais (SICAEV) da Secretaria Municipal de Saúde.

Obtivemos os dados dos anos de 1991-92 junto ao SICAEV, e analisamos as variáveis: sexo, procedência, idade materna, duração e tipo de gestação, peso ao nascer, hospital em que foi realizado o parto, ocorrência de necrópsia e causa básica da morte.

A variável procedência foi dividida em: residentes no Município de Ribeirão Preto (subdividida em setores: central, norte, sul, leste e oeste além do distrito de Bonfim Paulista) e residentes em outros municípios que não Ribeirão Preto e de procedência ignorada ou não declarada.

A idade materna foi agrupada segundo os intervalos: $<15,15-19 ; 20-24 ; 25-29$; 30-34; 35-39; 40- 44 e 45-49 anos, ignorada ou não declarada.

As variáveis sexo, duração e tipo de gestação e realização de necrópsia foram classificadas segundo o disposto na Declaração de Óbito.

$\mathrm{O}$ peso ao nascer foi disposto nos seguintes intervalos: menos de $1000 \mathrm{~g} ; 1000-1499 \mathrm{~g} ; 1500-1999 \mathrm{~g}$; 2000-2499g; 2500-2999g; 3000-3499g; 3500-3999g; igual ou superior a $4000 \mathrm{~g}$, ignorado ou não declarado.
O hospital responsável pelo atendimento à gestante foi catalogado.

A causa básica da morte foi classificada de acor-

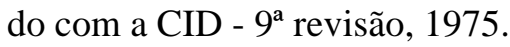

Os dados foram codificados e processados em microcomputadores 486, segundo o programa Epi-Info.

\section{RESULTADOS E DISCUSSÃO}

Foram registrados, nos cartórios do Município de Ribeirão Preto, 165 óbitos fetais, em 1991, e 134, em 1992, perfazendo 299, dos quais 148 do sexo masculino, 134 do feminino, 2 ignorados e 15 não declarados (Tabela I).

Nota-se que $49,5 \%$ foram do sexo masculino e $44,8 \%$ do feminino. A frequiência de procedentes de outros municípios $(39,5 \%)$, pode ser considerada alta, sendo decorrente do fato de ser, o município, pólo de atração para a assistência médica e hospitalar, em geral, como tem sido demonstrado por várias pesquisas. Em estudo realizado sobre a utilização de leitos hospitalares, Yazlle Rocha, $1972^{(5)}$, mostrou que 32,32\% eram ocupados por pacientes da região de Ribeirão Preto, $21,31 \%$ por procedentes de outras regiões e $46,37 \%$ por residentes neste município. A procura por assistência vem se mantendo ${ }^{(6,7)}$, principalmente para as afecções mais graves, tornando-se necessária, nos estudos de morbi-mortalidade, a classificação em residentes e não residentes, quando se visa traçar perfis para o município.

\begin{tabular}{|c|c|c|c|c|c|}
\hline \multirow{2}{*}{ Procedência } & \multicolumn{4}{|c|}{ Sexo } & \multirow[b]{2}{*}{ Tota } \\
\hline & M & $\mathrm{F}$ & $\lg ^{*}$ & $N D^{* *}$ & \\
\hline \multicolumn{6}{|l|}{ RibeirÃo PrEto } \\
\hline Centro & 1 & 2 & - & 1 & 4 \\
\hline Norte & 33 & 30 & - & 4 & 67 \\
\hline Sul & 6 & 6 & - & 2 & 14 \\
\hline Leste & 10 & 9 & - & 2 & 21 \\
\hline Oeste & 33 & 21 & 1 & 1 & 56 \\
\hline Bonfim Paulista & 1 & 3 & - & 1 & 5 \\
\hline OUtros MunicíPIos & 57 & 57 & 1 & 4 & 119 \\
\hline Ignorada & 5 & 3 & - & - & 8 \\
\hline Não Declarada & 2 & 3 & - & - & 5 \\
\hline TOTAL & 148 & 134 & 2 & 15 & 299 \\
\hline
\end{tabular}


A falta de preenchimento do quesito sexo (15 casos), na D.O., dificulta o conhecimento desta variável, quando se trabalha apenas com dados de Estatísticas Vitais, já que, para os óbitos fetais, não existe o registro de nascimento que poderia completar a informação.

Há grande variação na frequiência de óbitos fetais, segundo a divisão setorial do município, o que pode ser explicado pela heterogeneidade dos diferentes setores. Bonfim Paulista é um distrito localizado fora do perímetro urbano da cidade e conta com um número pequeno de habitantes. Os setores norte e oeste concentram grande parte da população do município e, em 1992, segundo dados do $\operatorname{SICAEV}^{(8)}, 76,1 \%$ dos nascidos vivos de Ribeirão Preto pertenciam a estes setores. A divisão do município em cinco setores e o distrito de Bonfim Paulista, utilizada pelo SICAEV, engloba, em cada um, população bastante heterogênea quanto ao nível sócio-econômico, o que torna difícil a análise pormenorizada tanto de um setor em particular quanto de um setor em relação a outros. No município, a população rural é muito pequena, correspondendo a menos de $2 \%$.

A maior frequiência de óbitos fetais foi verificada em mães nas faixas etárias de 20 a 24 anos e 25 a 29 anos, como se pode notar na Tabela II.

O número de Declarações em que a idade materna não foi assinalada (87), é bastante elevado, o que dificulta uma análise mais apurada da relação entre idade materna e óbito fetal. Apesar desta dificuldade, verifica-se que 36 dos nascidos mortos eram de mães adolescentes, sendo 3 em menores de 15 anos.
A gravidez em adolescentes vem aumentando no país, com percentuais diferentes nas várias regiões. Assim, em trabalho realizado em Pelotas, Tomasi; Barros \& Victora $^{(9)}$, encontraram uma variação de $15,3 \%$, em 1982, para 17,4\%, em 1993, de gestações em menores de 20 anos. Em Ribeirão Preto, Bettiol et al., 1992(10), estudando partos hospitalares, detectaram $14,1 \%$ em adolescentes, verificando que a menor frequiência ao pré-natal ocorreu neste grupo de mães, considerado o de maior risco de morbi-mortalidade materno-infantil.

$\mathrm{Na}$ Tabela III, acham-se assinalados o peso e duração da gestação dos óbitos fetais ocorridos no período.

\begin{tabular}{|c|c|c|}
\hline \multirow{2}{*}{ Idade Materna (anos) } & \multirow{2}{*}{$\begin{array}{l}\text { Nascidos } \\
n^{0}\end{array}$} & \multirow{2}{*}{$\begin{array}{c}\text { Mortos } \\
\%\end{array}$} \\
\hline & & \\
\hline$<15$ & 3 & 1,0 \\
\hline $15-19$ & 33 & 11,0 \\
\hline $20-24$ & 51 & 17,1 \\
\hline $25-29$ & 51 & 17,1 \\
\hline $30-34$ & 40 & 13,4 \\
\hline $35-39$ & 23 & 7,7 \\
\hline $40-44$ & 9 & 3,0 \\
\hline $45-49$ & 2 & 0,7 \\
\hline não declarado & 87 & 29,1 \\
\hline TOTAL & 299 & 100,0 \\
\hline
\end{tabular}

Tabela II - Óbitos Fetais, segundo idade materna, 1991-92

de óbitos fetais, Ribeirão Preto, 1991-9

\begin{tabular}{lccrrr} 
Peso (gramas) & \multicolumn{3}{c}{ Duração da Gestação } & Total \\
\cline { 2 - 5 } & $20-27$ semanas & 28 e + semanas & lg $^{*}$ & ND $^{* \star}$ & \\
\hline$<1000$ & 31 & 16 & 3 & 13 & 63 \\
$1000-1499$ & 4 & 16 & 4 & 8 & 32 \\
$1500-1999$ & 3 & 20 & 1 & 4 & 28 \\
$2000-2499$ & - & 18 & - & 4 & 22 \\
$2500-2999$ & 2 & 18 & - & 4 & 24 \\
$3000-3499$ & - & 11 & 1 & 2 & 14 \\
$3500-3999$ & - & 10 & - & - & 10 \\
4000 & - & 1 & - & 2 & 3 \\
Não Declarado & 12 & 32 & 1 & 58 & 103 \\
TOTAL & 52 & 142 & 10 & 95 & 299 \\
\hline
\end{tabular}

* Ignorado; **Não Declarado 
Nota-se que, em 103 Declarações $(34,4 \%)$, não há referência a peso ao nascer, apesar de que há balanças disponíveis para peso em todas as maternidades. Quanto à duração da gestação, em 142 casos $(47,5 \%)$, estava assinalado que era de 28 ou mais semanas, 52 perdas fetais $(17,5 \%)$ estavam entre 20 e 27 semanas e, em 105 casos $(35,3 \%)$, o quesito se encontrava em branco ou ignorado. Do total de perdas fetais com duração gestacional entre 20 e 27 semanas, em 31 casos, o peso é o esperado para a idade gestacional. Segundo os dados das Declarações de Óbito, pelo menos 142 casos foram de natimortos segundo a idade gestacional (28 semanas e mais) e 133, se tomarmos, como critério, a variável peso (1000 gramas ou mais).

Na fonte utilizada para o trabalho, não há informação sobre quando ocorreu a morte e portanto fica difícil tentar estabelecer associação entre peso e duração gestacional.

As percentagens de ignorado e não declarado podem ser consideradas altas, quando comparadas com dados obtidos por Baldijão \& Jorge ${ }^{(11)}$ para capitais da região Sudeste $(7,19 \%)$ e se aproximam mais dos das capitais da região Nordeste, apesar de a cobertura de serviço de saúde, no município, ser semelhante ao das capitais da região Sudeste. É importante assinalar que o dado referente a idade gestacional é mais fidedigno em áreas onde a cobertura dos serviços é melhor.

Em 140 casos $(46,8 \%)$, há referência à gestação única, em 14 (4,7\%), dupla, e apenas $1(0,3 \%)$ é referida como tripla. Este quesito encontra-se em branco em 139 declarações (46,5\%), o que dificulta a análise da informação. Pelo fato de esta ser uma variável de fácil obtenção, parece-nos que há descaso em seu preenchimento.

A Tabela IV apresenta a causa básica de morte e a frequiência de confirmação diagnóstica por necrópsia.

A hipoxia intra-uterina foi a causa básica mais freqüentemente assinalada, com 125 casos $(41,8 \%)$. No mesmo período, em estudo desenvolvido por Macharelli ${ }^{(12)}$, no Município de Botucatu, foi também a principal causa de natimortalidade $(35,7 \%)$. Todavia uma questão pode ser levantada: hipoxia intra-uterina pode ser comprovada em todos os casos, ou seria um diagnóstico inferido? Certamente, em muitos destes casos assinalados, de fato, deve ter havido hipoxia intra-uterina. Como determinar a precisão diagnóstica?

Alguns pesquisadores lançaram mão de outras classificações que não a CID, a exemplo de Zucoloto et al. ${ }^{(13)} \mathrm{e} \mathrm{Gurgel}^{(14)}$ que encontraram, em seus tra- balhos, aproximadamente $47 \%$ de causa de morte indeterminada. Fica claro, pela classificação utilizada pelos autores, que fizeram inclusive o cruzamento de dados de prontuários médicos e as respectivas necrópsias, que alguns dados relativos à causa indeterminada se sobreporiam aos da hipoxia, na classificação da CID.

Qual a classificação a se usar?

Utilizar causa indeterminada ao invés de hipoxia intra-útero, quando este é um dado apenas presumível, parece mais fidedigno.

Diante do exposto, entendemos ser esta uma questão que ainda necessita de maior discussão.

Outro achado importante se refere às afecções maternas não obrigatoriamente relacionadas à gravidez atual (CID-760) que, somadas às complicações maternas da gravidez (CID-761) e complicações da placenta, cordão e membranas (CID-762), perfazem 50,2\% das causas básicas de morte. Excluídas estas e hipoxia intra-útero (CID-768) restam apenas 8,4\% de casos, distribuídos pelas demais causas (Tabela IV).

Em trabalho desenvolvido no município, Goldani ${ }^{(15)}$, tendo encontrado associação entre morte precoce de crianças e natimortalidade, chama a atenção para a relação entre o passado obstétrico, mórbido das mães e a qualidade da assistência médica à gestação e ao parto. Assim, o conhecimento das causas de natimortalidade pode servir como indicativo de cuidados específicos no atendimento pré-natal, principalmente para as gestantes com história prévia de perda fetal. Para que isto se efetive, é importante um melhor preenchimento, por parte dos médicos, das informações na Declaração de Óbito, utilizando mais os recursos diagnósticos disponíveis no município, como a realização de necrópsias, nos casos não esclarecidos, bem como uma maior divulgação dos achados para quem realiza o atendimento.

A maioria das necrópsias foram efetuadas em perdas fetais ocorridas no Hospital das Clínicas, fato este que denota a maior preocupação deste hospital em esclarecer os diagnósticos, por tratar-se de um hospital universitário.

Ocorreu, no período, uma morte fetal e materna por acidente de trânsito.

O coeficiente médio por perdas fetais, para o biênio 91-92, foi de 19,3\% nascidos vivos mais nascidos mortos. Quando excluídos os casos 
procedentes de outros municípios e os que não se enquadram na idade gestacional de 28 e mais semanas, ou seja, quando se calcula o coeficiente de natimortalidade, o valor cai para 8,9\% nascidos vivos mais nascidos mortos. Este coeficiente pode ser considerado baixo, quando comparado com os de outros estudos realizados no Brasil, como o de Victora et al. ${ }^{(16)}$, em Pelotas, 1982, que encontrou $16,1 \%$.

Quanto à realização de necrópsias, o quesito encontra-se sem preenchimento, em 117 Declarações, o que pode ser considerado como um indício da não-realização da mesma.

\section{CONCLUSÕES}

Dos óbitos fetais registrados no período de estudo, 39,8\% eram procedentes de outros municípios, configurando Ribeirão Preto como pólo de assistência à saúde. Destas perdas, 47,5\% tinham idade gestacional de 28 ou mais semanas, configurando-se como natimortos.

Apesar da maior frequiência em mães entre 20 e 29 anos, em $29,1 \%$ não se encontrava declarada a idade materna.

A anoxia intra-uterina foi a causa mais importante de morte $(41,8 \%)$, seguida de complicações de placenta, cordão e membranas $(25,4 \%)$.

A confirmação diagnóstica por necrópsia ocorreu em $58 \%$ das perdas fetais.

Chama a atenção a falta do preenchimento de vários quesitos, muitos deles, de fácil obtenção por quem está declarando o óbito. Este fato inviabiliza análises mais apuradas dos dados.

Sugere-se que trabalhos devem ser feitos junto aos médicos para a melhoria do preenchimento das Declarações de Óbito.

Tabela IV - Perdas Fetais segundo causa básica e confirmação diagnóstica por necrópsia, Ribeirão Preto, 1991-92

\begin{tabular}{|c|c|c|c|c|c|}
\hline \multicolumn{2}{|r|}{ Causa básica } & \multicolumn{3}{|c|}{ Necrópsia } & \multirow{3}{*}{$\begin{array}{l}\text { Total } \\
125\end{array}$} \\
\hline & & \multirow{2}{*}{$\begin{array}{r}\text { Sim } \\
83\end{array}$} & \multirow{2}{*}{$\begin{array}{c}\text { Não } \\
5\end{array}$} & \multirow{2}{*}{$\begin{array}{c}\mathrm{ND}^{*} \\
37\end{array}$} & \\
\hline 768 & Hipoxia intra-uterina & & & & \\
\hline 762 & $\begin{array}{l}\text { Complicações da placenta, cordão e membranas que } \\
\text { afetam o feto }\end{array}$ & 30 & 4 & 42 & 76 \\
\hline 760 & Afecções maternas da gravidez, que afetam o feto & 27 & 1 & 10 & 38 \\
\hline 761 & Complicações maternas que afetam o feto & 19 & - & 17 & 36 \\
\hline 759 & Anomalias congênitas não especificadas & 3 & - & 3 & 6 \\
\hline 799 & $\begin{array}{l}\text { Outras causas mal definidas de morbidade e morta- } \\
\text { lidade }\end{array}$ & 1 & - & 3 & 4 \\
\hline 779 & $\begin{array}{l}\text { Outras afecções e as mal definidas originadas no pe- } \\
\text { ríodo perinatal }\end{array}$ & 2 & - & 1 & 3 \\
\hline 740 & Anencefalia & 2 & - & 1 & 3 \\
\hline 765 & $\begin{array}{l}\text { Transtornos relacionados à gestação de curta duração } \\
\text { e baixo peso ao nascer, de tipo não especificado }\end{array}$ & 1 & - & 1 & 2 \\
\hline 763 & $\begin{array}{l}\text { Outras complicações do trabalho de parto ou do parto } \\
\text { que afetam o feto }\end{array}$ & 1 & - & - & 1 \\
\hline 764 & Crescimento fetal retardado e má nutrição fetal & 1 & - & - & 1 \\
\hline 771 & Infecções específicas do período perinatal & - & - & 1 & 1 \\
\hline 772 & Hemorragia fetal e neonatal & 1 & - & - & 1 \\
\hline 773 & Doença hemolítica do feto, devida à isoimunização & 1 & - & - & 1 \\
\hline \multirow[t]{2}{*}{-} & Prejudicado & - & - & 1 & 1 \\
\hline & TOTAL & 172 & 10 & 117 & 299 \\
\hline
\end{tabular}

* não declarada 
SARDAS R \& MANÇO ARX. Fetal mortality in Ribeirão Preto City, Brazil, 1991 and 1992. Medicina, Ribeirão Preto, 30: 508-513, oct./dec. 1997.

ABSTRACT: A total of 299 fetal death occured in the Municipality of Ribeirão Preto in 1991/92, 165 in 1991 and 134 in 1992, 148 of which were boys and 134 were girls, 21 of unknown sex, and 15 not declared.

The fetal death from other municipalities correspondend to $39,8 \%$ of this total. Birth weight was not declared on 103 Declarations.

The durantion of gestation was 28 or more weeks in 142 cases $(47,5 \%), 20$ to 27 weeks in 52

$(17,4 \%)$, and this information had not been filled or was marked as unknown in 105 cases $(35,3 \%)$. A single gestation was pointed out in $46,8 \%$ of cases and was not declared in $46,5 \%$. Intrauterine hypoxia was indicated as underying causa in $41,8 \%$ of cases.

UNITERMS: Fetal Death. Fetal Mortality.

\section{REFERÊNCIAS BIBLIOGRÁFICAS}

1 - MANUAL DE CLASSIFICAÇÃO ESTATÍSTICA INTERNACIONAL DE DOENÇAS, LESÕES E CAUSAS DE ÓBITOS, 9ª revisão, 1975. OMS, Genebra, 1978.

2 - HECKMANN IC et al. Análise do preenchimento de declarações de óbitos em localidade do Estado do Rio Grande do Sul (Brasil) 1987. Rev Saúde Pública 23: 292-297,1989.

3 - MENDONÇA EF; GOULART EMA \& MACHADO JAD. Confiabilidade da declaração de causa básica de mortes infantis em uma região metropolitana do sudeste do Brasil. Rev Saúde Pública 28: 385-391, 1994.

4 - LAURENTI R et al. Estatísticas de saúde. EPU-EDUSP, São Paulo, 1985.

5 - YAZLLE ROCHA JS. Estudo da utilização de leitos hospitalares gerais do Município de Ribeirão Preto, pela população do município, no ano de 1972. Tese de Doutorado, Faculdade de Medicina de Ribeirão Preto da USP. Ribeirão Preto, 1974.

6 - ARANTES GR; XAVIER AR \& ROLANDO E. Uso da invasão e evasão de óbitos para identificar pólos de atração médico-assistencial: estudo realizado em uma Divisão Regional de Saúde de São Paulo (Brasil) Rev Saúde Pública 15: 20-37, 1981.

7 - YAZLLE ROCHA JS; SIMÕES BJG \& FORSTER AC. Estudos de demanda e utilização de serviços de saúde: uma experiência regional. Medicina, Ribeirão Preto 26: 636-642, 1993.

8 - SECRETARIA MUNICIPAL DA SAÚDE. Sistema de Coleta e Análise de Estatísticas Vitais. Ribeirão Preto, Boletim no 1 ano II, 1992.
9 - TOMASI E; BARROS FC \& VICTORA CG. As mães e suas gestações: comparações de duas coortes de base populacional no sul do Brasil. Cad Saúde Pública, Rio de Janeiro 12: 21-25, 1996. Supl 1.

10 - BETTIOL $\mathrm{H}$ et al. Atenção médica à gestação e ao parto de mães adolescentes. Cad Saúde Pública, Rio de Janeiro 8: 404-413, 1992.

11 - BALDIJÃO MF de A \& JORGE MHP de M. Óbitos fetais no Brasil no período de 1980 a 1985: análise de informação disponível no Ministério da Saúde. Centro da OMS para a Classificação de Doenças em Português, São Paulo, 1989. (Série Divulgação, 4)

12 - MACHARELLI CA. Mortalidade perinatal em Botucatu, SP, 1991/1992. Dissertação de Mestrado, Faculdade de Medicina de Ribeirão Preto da USP, Ribeirão Preto, p. 1-109, 1995.

13 - ZUCOLOTO S et al. Estudo do comportamento das taxas de mortalidade fetal, perinatal e neonatal em um Hospital Universitário. III. Evolução do perfil patológico das causas básicas de morte, obtido em necrópsia. J Pediatr 64: 299-305, 1983.

14 - GURGEL RQ. Estudo da evolução da mortalidade perinatal e neonatal, por necrópsias no HCFMRP-USP no período de 1973 a 1982. Dissertação de Mestrado, Faculdade de Medicina de Ribeirão Preto da USP. Ribeirão Preto, p.1-117, 1986.

15 - GOLDANI MZ. Mortalidade infantil em Ribeirão Preto: comparação entre duas coortes em um intervalo de 15 anos, 1979/94. Tese de Doutorado, Faculdade de Medicina de Ribeirão Preto da USP. Ribeirão Preto, p.1-153, 1997.

16 - VICTORA CG; BARROS FC \& VAUGHAN JP. Epidemiologia da desigualdade. Editora Hucitec, São Paulo, 1989. 